Advanced Computing: An International Journal ( ACIJ ), Vol.4, No.3, May 2013

\title{
PERFORMANCE EVALUATION OF ONTOLOGY AND FUZZYBASE CBIR
}

\author{
Tajman sandhu (Research scholar) \\ Department of Information Technology \\ Chandigarh Engineering College, Landran, Punjab, India \\ yuvi_tajeyahoo.com \\ Parminder Singh(Assistant Professor) \\ Department of Information Technology \\ Chandigarh Engineering College, Landran, Punjab, India \\ Singh.parminder06@gmail.com
}

\begin{abstract}
IN THIS PAPER, WE HAVE DONE PERFORMANCE EVALUATION OF ONTOLOGY USING LOW-LEVEL FEATURES LIKE COLOR, TEXTURE AND SHAPE BASED CBIR, WITH TOPIC SPECIFIC CBIR.THE RESULTING ONTOLOGY CAN BE USED TO EXTRACT THE APPROPRIATE IMAGES FROM THE IMAGE DATABASE. RETRIEVING APPROPRIATE IMAGES FROM AN IMAGE DATABASE IS ONE OF THE DIFFICULT TASKS IN MULTIMEDIA TECHNOLOGY. OUR RESULTS SHOW THAT THE VALUES OF RECALL AND PRECISION CAN BE ENHANCED AND THIS ALSO SHOWS THAT SEMANTIC GAP CAN ALSO BE REDUCED. THE PROPOSED ALGORITHM ALSO EXTRACTS THE TEXTURE VALUES FROM THE IMAGES AUTOMATICALLY WITH ALSO ITS CATEGORY (LIKE SMOOTH, COURSE ETC) AS WELL AS ITS TECHNICAL INTERPRETATION.
\end{abstract}

\section{KEYWORDS}

CBIR; fuzzyminmax; recall; precision; Texel; texture

\section{INTRODUCTION}

Content Based Image Retrieval system is a system in which the retrieval is based on the content as well as linked information of the image which is having mathematical value in nature. Contentbased image retrieval (CBIR) [1] also recognized as query by image content (QBIC) and contentbased visual information retrieval (CBVIR). There is a increasing interest in CBIR since of the limitations inherent in metadata-based systems and the great choice of possible uses for efficient image retrieval.CBIR systems have been developed, however the problem of retrieving images on the foundation of their pixel content remains largely unexplained.

\section{DIFFERENT IMPLEMENTATIONS OF CBIR}

Different implementations of CBIR make use of different types of client queries. 


\subsection{Query by example}

Query by example is a query technique with the purpose of involves providing the CBIR system with an example image that it will then base its search upon. .This query technique removes the difficulties that can arise when trying to explain images with words.

\subsection{Query by Semantic objects}

The perfect CBIR system from a customer viewpoint would involve what is referred to as semantic retrieval, where the customer makes demand like "find pictures of smooth texture". This type of open-ended task is very hard for computers to perform. Current CBIR systems therefore generally make use of lower-level features [2] like texture, color, and shape even though some systems take advantage of very common higher-level features [3] like faces. Not every CBIR system is generic. Various systems are designed for a particular domain or topic specific e.g. shape matching can be used for judgment parts inside a CAD-CAM database.

\subsection{Other query methods}

additional query methods consist of browsing for example images, navigating customized/hierarchical categories, querying by image part (rather than the entire image), querying by multiple example images, querying by visual drawing, querying by direct specification of image features, and multimodal queries (e.g. combining touch, voice, etc.).

\section{BUILT CBIR}

\subsection{Color}

Color standards similar to $(255,111,40)$ represents color features by Computing distance measures which is based on color resemblance that is achieved by computing a color histogram for each image which identifies the proportion of pixels within an image holding exact values (that humans express as colors). Present research is attempting to segment color proportion by region and by spatial relationship among a number of color regions. Examining images based on the colors they hold is one of the most broadly used techniques for the reason that it does not depend on image size or orientation. Color searches will usually engage comparing color histograms, despite the fact that this is not the only technique in put into practice.

\subsection{Texture}

Texture [9] standards similar to $(0.2,0.3$, and 0.4$)$ of energy measures the visual patterns in images as well as how they are spatially defined. Textures are represented by means of texels [4] which are then positioned into a numeral of sets, depending on how many textures are detected in the image. These sets not only define the texture, however also where in the image the texture is situated.Texture is a complicated theory to represent. The identification of accurate textures in an image is achieved primarily by modeling texture as a two-dimensional gray level variation. The comparative intensity of pairs of pixels is computed such that degree of contrast, regularity, coarseness and directionality possibly will be predictable. On the other hand, the problem is in identifying patterns of co-pixel variation as well as associating them with particular classes of textures such as silky, or irregular. 


\subsection{Shape}

Shape does not refer to the shape of an image but to the shape of a particular region that is to say being sought out. Shapes will frequently be determined first applying segmentation or edge detection to an image. Additional methods like use shape filters to identify particular shapes of an image. In a few cases correct shapes recognition will require human interference for the reason that methods like segmentation are very complicated to completely automate.

\section{Evaluation of CBIR}

There are many CBIR available, but how do we know, which one is performing really fine, which one gives the best quality. The results both in terms of images and the associated annotated information need to be addressed and justified for the said system for performance. For SLR [5] technique, we come to understand the most CBIR are evaluated based on recall and precision Evaluation of retrieval performance plays a very important role in image retrieval. There are many different methods used for performance evaluation of the system. The most common evaluation measures used in image retrieval systems are Precision [6] and Recall [6], usually presented as precision versus recall graph (PR) graph.

Precision: The ability to retrieve top-ranked images that are mostly relevant

$$
\text { Precision Value }=\frac{\text { Total numbar of ralavant rasults ratriaved }}{\text { Tokal rasults takan out }}
$$

Recall: The ability to the search and find all of the relevant items in the database.

Recall Value $=\frac{\text { Total number of relevant results retrieved }}{\text { Total number of images in the database }}$

In this research paper we intend to find which CBIR is better performing if multiple features are used, or a system that uses single feature based on color, texture, shape etc., therefore, we propose following system as compared to Construction of Image Ontology using Low-Level features for Image Retrieval system.

The flowchart of the proposed algorithm is as follows:-

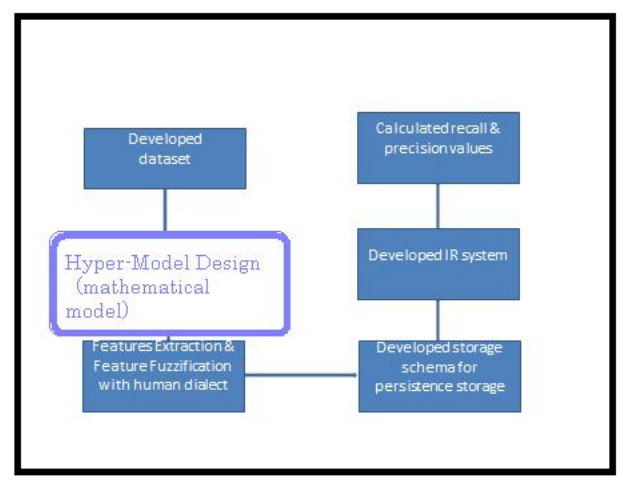

Fig 1 Flowchart of the Proposed Algorithm 
Following are the steps:-

1. Development of a representative data set of textured images.

2. Study of various linguistic descriptors (Fuzzy Sets[7]) based on the domains of textured images with degree of entropy, homogeneity, contrast, cluster shade, Autocorrelation and with interpretation

3. Use of Fuzzy Sets collected in step 2and development and annotation of texture features sets for the said data set developed in step 1 .

4. Development of a storage schema which maps the Hyper Model and texture feature annotation sets developed in step 3.

5. Development of an interface for storing the highly textured images information w.r.t. previous steps in the database.

6. Development of a structured query based on which information of texture images can be retrieved.

7. Development of an application for running the queries developed in step 6 .

8. Calculation of precision and recall values for design and implementing hyper real model by reducing semantic gap using image retrieval system

9. Comparison of the planned system with other systems.

This system architecture describes the flow of processes involved in construction of Image Ontology using Color, Texture and Shape from the input images present in the database.

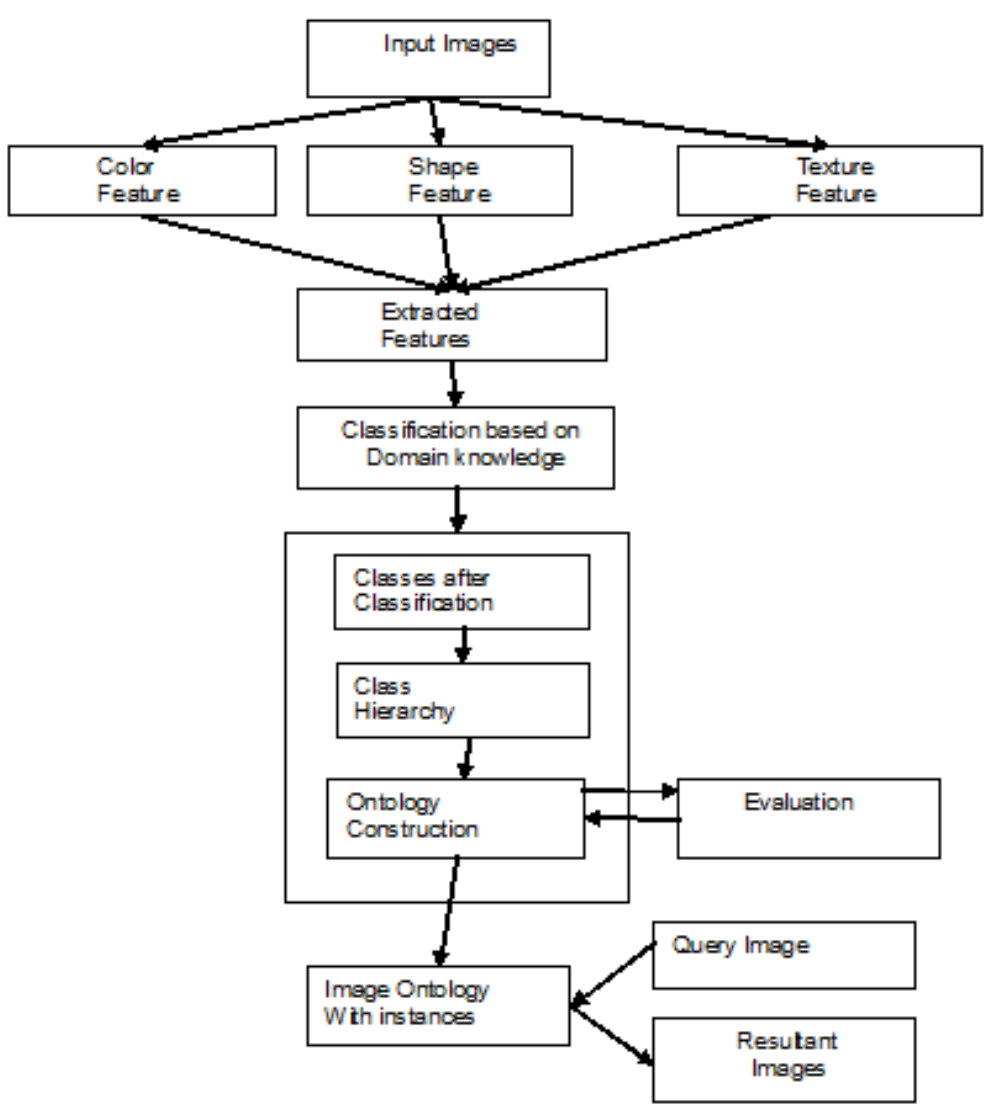


Steps involved in previous algorithm are

1. Images are input from the data base

2. Then the image ontology based upon color, texture and shape is extracted

3. Then classification is done in which grouping is done according to some external criteria

4. This Classification is done manually followed by class hierarchy construction

5. This system uses Seven Step Methodology"

It consists of the following steps,

- Determine the domain and scope of ontology

- Reusing existing ontology, if any

- Enumerate important terms in the ontology

- Define the classes and class hierarchy

- Define the properties of classes (slots or roles)

- Define the facets of the slots (role restrictions)

- Create Instance

6. Then query images are matched with data base images and the most similar images are retrieved.

\section{Performance evaluation of both systems}

Following parameters are used to compute the results and recall values for both the system. These values are fed into formulas as described above. From where value of recall and precision is calculated.

Table 1.example query and its calculation

\begin{tabular}{|c|c|c|}
\hline 1. & $\begin{array}{c}\text { Total no. of images in } \\
\text { database }\end{array}$ & 100 \\
\hline 2. & $\begin{array}{c}\text { Total number of result } \\
\text { shown }\end{array}$ & 70 \\
\hline 2. & $\begin{array}{c}\text { Total number of correct } \\
\text { relevant results ( for } \\
\text { 'smooth texture' query) }\end{array}$ & 12 \\
\hline 3. & $\begin{array}{c}\text { Total number of Relevant } \\
\text { Image results in the } \\
\text { database but not shown } \\
\text { Total no. of Irrelevant } \\
\text { Images results }\end{array}$ & 12 \\
\hline 4. & Recall & 0.48 \\
\hline 5. & Precision & 0.685 \\
\hline
\end{tabular}

Table 2.Recall precision values [8]

\begin{tabular}{|l|l|}
\hline Recall value & Precision value \\
\hline 0.2 & 1 \\
\hline 0.4 & 0.83 \\
\hline 0.6 & 0.66 \\
\hline 0.8 & 0.66 \\
\hline
\end{tabular}


Table 3.Recall precision values of proposed system

\begin{tabular}{|l|l|}
\hline Recall value & Precision value \\
\hline 0.2 & 0.83 \\
\hline 0.48 & 0.685 \\
\hline 0.6 & 0.705 \\
\hline 0.8 & 0.88 \\
\hline
\end{tabular}

Recall precision graph

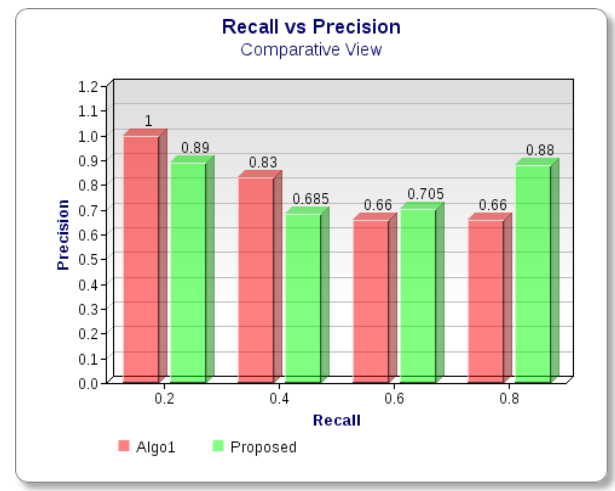

\subsection{Interpretation of graph}

It is apparent from the graph that the quality of the results is increasing in terms of precision, as the recall value increases. This, however, reflects that the queries are specific to topic and get broader in their scope within same domain, due to which recall also remain highs.

\section{Conclusion}

The previous algorithm is using multiple low level features, which includes color, texture and shape and based on ontology, they have done manual classification and annotation, however, we have developed a system that automatically extracts texture information, identify its degree and interpretation in technical words that are understood by humans easily, this reduces the semantic gap, and it is apparent from the table[2] and [3] as well as from the bar graph that the focused ,topic specific, domain specific CBIR produce better results in terms of proportions of relevant results when information is retrieved from it.

\section{Future scope}

Our work can be further extended by understanding the semantic of other texture properties of the images and also by mapping technical and scientific laws that give the values of texture features likeCluster Prominence, Dissimilarity, Variance, Maximum probability, Inverse difference normalized which have not been used in current work. 


\section{Acknowledgment}

I degree my thanks to all who helped me in successful completion of this current research work. I would like to admit the assistance and support acknowledged from Er. Parminder Singh, who gave me fruitful opportunity to expose our knowledge by doing this research work.

\section{References}

[1] Ricardo da Silva Torres and Alexandre Xavier Falcão ," Content-Based Image Retrieval: Theory and Applications"

[2] D. Brahmi," Improving CBIR Systems by Integrating Semantic Features”, University of Western Ontario, London, Ontario, Canada ,May 17-May 19

[3] Ying Liu, Dengsheng Zhang, Guojun Lu, and Wei-Ying Ma," A survey of content-based image retrieval with high-level semantics", Pattern Recognition, Volume 40, Issue 1, January 2007, Pages 262-282

[4] Todorovic, S.," Texel-based texture segmentation/"Computer Vision, 2009 IEEE 12th International Conference on Sept. 29 2009-Oct. 2 2009, pp. 841 - 848J. Clerk Maxwell, A Treatise on Electricity and Magnetism, 3rd ed., vol. 2. Oxford: Clarendon, 1892, pp.68-73.

[5] VIJAY V. RAGHAVAN and GWANG S. JUNG," A Critical Investigation of Recall and Precision as Measures of Retrieval System Performance" University of Southwestern Louisiana And PETER BOLLMANN Technische Universitat Berlin

[6] Ricardo da Silva Torres and Alexandre Xavier Falcão ," Content-Based Image Retrieval: Theory and Applications"

[7] Didier DuBois, Henri M. Prade,'Fundamentals of Fuzzy Sets"

[8] Gowsikhaa.D Abirami.S and Baskaran.R," Construction of Image Ontology using Low-Level features for Image Retrieval" 2012 International Conference on Computer Communication and Informatics (ICCCI -2012), Jan. 10 - 12, 2012, Coimbatore, INDIA

[9] Tajman sandhu and parminder singh,"domain specific CBIR for highly textued images " Computer Science \& Engineering: An International Journal (CSEIJ), Vol. 3, No. 2, April 2013 